International Journal of Nano Studies \& Technology (IJNST) ISSN:2167-8685

\title{
Techniques Used For Development of Aqueous Polymeric Dispersion
}

Research Article

Singh $\mathrm{S}^{1}$, Mittal A ${ }^{1}$, Murtuja S ${ }^{2}$, Bajaj $\mathrm{UK}^{3}$, Alam $\mathrm{S}^{*}$

${ }^{1}$ Department of Pharmaceutics, KIET School of Pharmacy, Ghaziabad.

${ }^{2}$ Department of Pharmaceutical Chemistry, KIET School of Pharmacy, Ghaziabad.

${ }^{3}$ Department of Pharmacology, KIET School of Pharmacy, Ghaziabad.

Abstract
Recent trends in pharmaceutical industry are development of coating methods which overcomes the various disadvantages associ-
ated with solvent based coatings. Water insoluble polymers have largely superseded the use of organic solvents for applying coatings.
Aqueous polymer dispersions (APD) are preferred on environmental and safety grounds. APD offer several advantages over polymers
dissolved in organic solvents including lower spraying viscosities, higher solids loading, higher spray rates, no solvent environmental,
toxicity, or flammability issues, and reduced energy requirements relative to Aqueous polymer solutions. There are a wide range of
water insoluble polymers includes Acrylates: Eudragit, Cellulose: Ethyl cellulose, Vinylics: Polyvinyl acetate phthalate, Cellulosic esters:
cellulose acetate phthalate (CAP) \& many more which provide a great research platform to develop an Aqueous polymeric dispersion
(APD). This article is mainly concerned with the theoretical \& practical aspects of the techniques/steps \& research scope for the
development of APD.
Key Words: Aqueous Polymeric Dispersion (APD); Emulsification Solvent Evaporation; Nanotechnology; Spontaneous Emulsifica-
tion Solvent Diffusion (SESD)

\author{
*Corresponding Author: \\ Sanjar Alam \\ Department of Pharmaceutics, KIET School of Pharmacy, Ghaziabad, \\ U.P-201206, India. \\ Tel: +91- 9891674226. \\ E-mail: sanjaralam10@gmail.com
}

Accepted: February 05, 2014

Accepted: April 04, 2014

Published: April 07, 2014

Citation: Alam S, et al. (2014). Techniques Used For Development of Aqueous Polymeric Dispersion, Int J Nano Stud Technol, 03(01), 40-44. doi: http://dx.doi.org/10.19070/2167-8685-140008

Copyright:Alam $\mathbf{S}^{\odot}$ 2014. This is an open-access article distributed under the terms of the Creative Commons Attribution License, which permits unrestricted use, distribution and reproduction in any medium, provided the original author and source are credited.

\section{Introduction}

Polymers have changed our day-to-day lives over the past several decades. Polymers represent an important constituent of pharmaceutical dosage form . They are main tool to control the drug release rate from the formulations. Infact the formulation and clinical performance of dosage form depend on the physicochemical property of the polymer used in the formulations [1] .They are used as taste-masking agent, stabilizer, and protective agent in oral drug delivery. Polymers can bind the particles of a solid dosage form and also change the flow properties of a liquid dosage form [2].

Coating with water insoluble polymers is an important technique for targeted or controlled release formulations and the tastemasking of drugs, during which the aqueous polymer dispersions are widely used as an alternative to an organic polymer coating system [3].Water soluble polymers are always being in good demand because of their certain advantages over organic solvents with respect to ecological, toxicological and manufacturing safety concern, high cost of organic solvents, solvent toxicity [4].

Aqueous polymeric dispersions, latexes, or pseudo latexes are all colloidal systems in which high molecular weight polymers are homogeneously dispersed in submicron sizes with the aid of surfactant(s) and other stabilizing agents. aqueous dispersion containing hydrophobic material that are useful as reduced volatile content may be used as a coating, resin,or an additive [5].

\section{APD: A Colloidal System}

Colloidal system is a two phase system that comprises a dispersed phase and a dispersion medium.

The dispersed phase in latex comprises spherical polymer particles, usually with average diameter of 200-300 $\mathrm{nm}$. The dispersion medium is water that contains various water- soluble compounds the properties of the aqueous phase are determined by the type and concentration of dissolved compounds (simple electrolytes, surfactants and polyelectrolytes) and $\mathrm{pH}[6]$.

\section{Types of Aqueous Polymeric Dispersion}

Aqueous polymeric dispersions that are widely used by the pharmaceutical industry are manufactured using different processes and different starting materials.

They are classified into:

Latex: Latexes are aqueous polymeric dispersions produced by emulsion polymerization. The homogeneous and heterogeneous 
Table 1.Different types of emulsification

\begin{tabular}{|c|c|c|c|}
\hline Properties & Direct & Inversion & Self \\
\hline Definition & $\begin{array}{l}\text { The liquid polymer in a vola- } \\
\text { tile organic solvent is emul- } \\
\text { sified in water that contain } \\
\text { emulsifier \& stabilizer by us- } \\
\text { ing conventional emulsifica- } \\
\text { tion method [7] }\end{array}$ & $\begin{array}{l}\text { The liquid polymer in an organic solvent is com- } \\
\text { pounded with long fatty acid with conventional rub- } \\
\text { ber mixing equipment. Water in polymer inverts to } \\
\text { polymer in water emulsion. } \\
\text { phase inversion is complete, which means that all wa- } \\
\text { ter molecules in the system are transformed to be } \\
\text { continuous phase at the phase inversion point [8] }\end{array}$ & $\begin{array}{l}\text { Polymer molecules are modi- } \\
\text { fied chemically by the intro- } \\
\text { duction of basin or acidic } \\
\text { group \&self emulsification } \\
\text { occurs [6] }\end{array}$ \\
\hline Droplet size & $1 \mu \mathrm{m}$ or larger & $0.8-1 \mu \mathrm{m}$ or larger & Small as $0.1 \mu \mathrm{m}$ \\
\hline $\begin{array}{l}\text { Ease of } \\
\text { preparation }\end{array}$ & Easy to prepare & Require great care and control & Not easy to prepare \\
\hline Cost & Economic & Expensive & Expensive \\
\hline example & Eudragit & Polyacrylamide \& its co- polymer [9] & \\
\hline
\end{tabular}

nucleation models have been proposed to describe such processes.

As a direct outcome of the manufacturing process, latex may contain not only polymers, but also surfactants, as well as traces of initiators and monomers that must be eliminated as completely as possible from the lattice used in pharmaceutical applications

Pseudo latexes: Pseudo latexes are prepared by dispersion of the bulk polymer in an aqueous medium. The pseudolatex dispersion, a coating formulation often includes plasticizers to enhance the flexibility of the film and facilitate polymer sphere coalescence, antiadherents to prevent substrate agglomeration during both the coating process and storage, surfactants to promote spreading of the atomized droplets own the substrate surface, and pigments. The addition of other excipients can significantly impact the physical stability of the dispersion, drug release, and film quality.

An APD can be prepared in two different forms:

a. Ready to use: milky white liquid

b. Freeze dried / spray dried: powdered

\section{Different Techniques Used In APD}

Water insoluble polymers can be converted into aqueous dispersions by emulsion polymerization, emulsion solvent evaporation, phase inversion, or solvent change method

1. Emulsification polymerization

- Direct Emulsification/ emulsion solvent evaporation

- Self Emulsification

- Phase Inversion Emulsification or Solvent change method

2. Nanotechnology

3. $\mathrm{pH}$ modification

4. Phase separation/ coacervation

\section{- Emulsification Technique}

\section{Development of APD}

An APD can be prepared by using any of technologies- emulsification, nanotechnology, $\mathrm{pH}$ modification etc. under three major steps:

1. Development of spray dried aqueous polymeric dispersion

2. Development of polymeric films

3. Performance characterization of developed APD

\section{Development Of Spray Dried Aqueous Polymeric Dis- persion}

1.1 Selection of organic phase

1.2 Selection of emulsifier

1.3 Selection of stabilizer

1.4 Formulation of aqueous polymeric dispersion

- Solvent evaporation technique

1.5 Characterization of prepared aqueous dispersion

- Organoleptic

- Physical

- Chemical interaction

- Solid content

1.6 Spray drying

1.7 Stability study

\section{Development Of Polymeric Films}

After formation of APD, free films are prepared by diluting the dispersion in distilled water \& cast in Teflon mould. Then films are peeled off. Characterization of films is done

\section{Mechanism of film formation}

The film-coating process requires a delicately balanced environment. Formation of an acceptable layer of film on the substrate requires the following:

1. Formation of appropriate-size droplets

2. Contact of these droplets with the substrate

3. Spreading and coalescence of the droplets

4. Evaporation of the solvent

\section{Performance Characterization Of Developed APD}

3.1 Matrix tablets for sustained or controlled delivery.

3.2 Film coating: Tablet coating, taste masking, microencapsulation, stability enhancement 
Figure 1. Flow diagram for the development of APD using emulsification technology

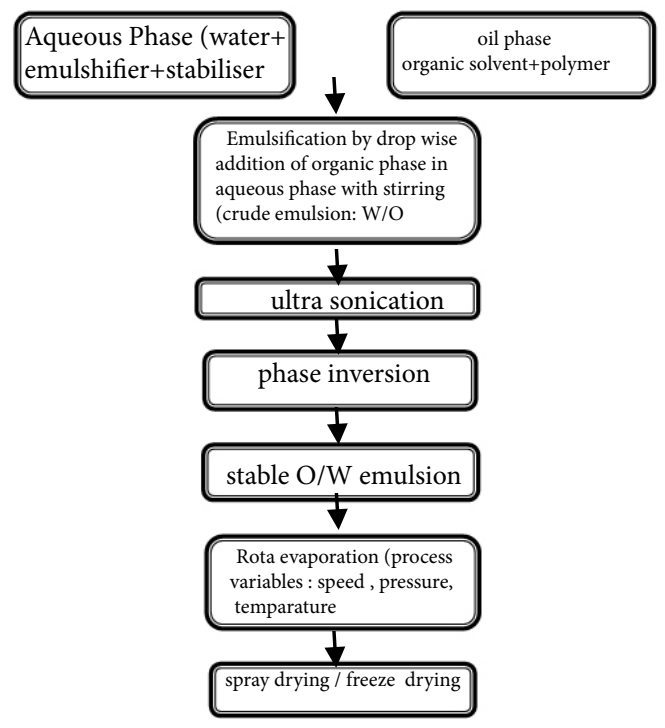

Figure 2. Flow diagram of film formation

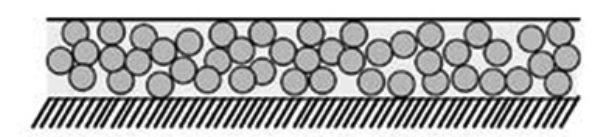

Aqueous Dispersion deposited on surface

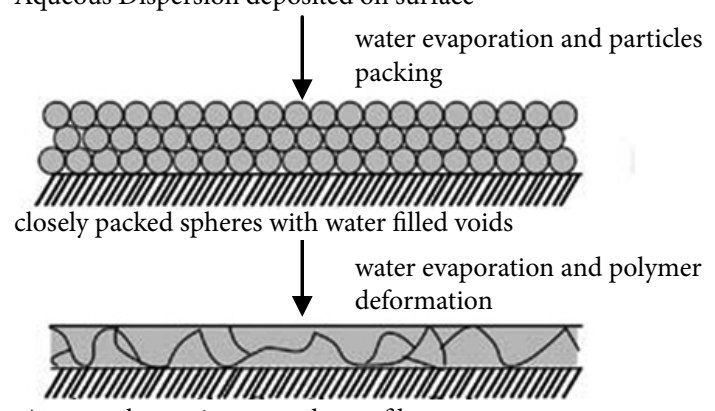

Apparently continuous polymer film

water diffusion and polymer interdiffusion
Figure 3. Preparation of polymer nanoparticle

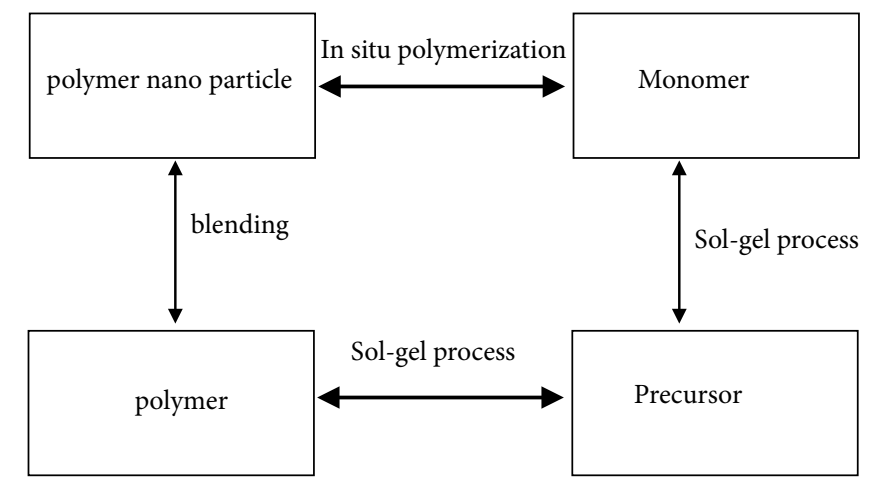

\section{- Nanotechnology}

In this methodology polymeric solution in a water immiscible organic solvent is mixed with water having surfactants \& nanospheres are formed.

- Three general approaches to prepare polymer nanoparticle:

\section{Sol-Gel Process}

This is a very useful novel reinforcement technique, which can generate reinforcing particles within a polymer matrix. Moreover, these novel hybrid sol-gel materials are normally nanocomposites.

\section{In Situ Polymerization}

The process of in situ polymerization involves three continuous steps:

i. First, the nanoscale additives are pretreated with appropriate surface modifiers ii. Second, dispersion of modified additives into monomer(s).

iii. Third, bulk or solution polymerization.

Nanocomposites are formed in situ during the polymerization [10]. These provide the nanospheres of size range 50-500 $\mathrm{nm}$ [11].

e.g. - Synthesis of Vinyl Polymer, Silica Colloidal Nanocomposites via Aqueous Dispersion Polymerization.

2-hydroxypropyl methacrylate [HPMA] is copolymerized with 4-vinylpyridine [4VP] using ammonium persulfate in the presence of an ultrafine silica sol. 4VP is used as an auxiliary in these syntheses; the strong interaction of this basic monomer with the acidic surface of the silica particles is essential for successful nanocomposite particle formation [12].

\section{Salting Out}

Polymeric nanoparticle are prepared using an emulsion technique involving a salting-out process which avoids surfactants and chlo- 
Figure 4.Preparation procedures of the original SESD method and the modified-SESD method

SESD method

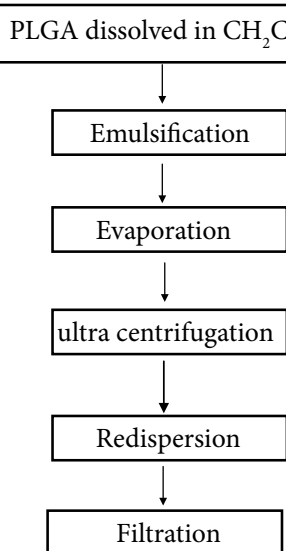

Modified SESD method

PLGA dissolved in alcohol/ acetone

Dispersion

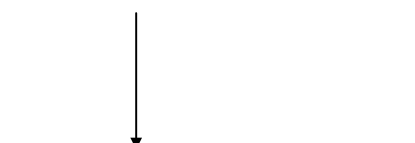

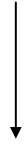

ultra filteration

L

rinated solvents. The preparation method consists of adding an electrolyte-saturated or a non-electrolyte-saturated aqueous solution containing poly (vinyl alcohol) (PVAL) as a viscosity-increasing agent and stabilizer to an acetone solution of polymer under continuous stirring. The saturated aqueous solution prevents acetone from mixing with water by a salting-out process. After the preparation of an oil-in-water emulsion, water is added in a sufficient amount to allow complete diffusion of acetone into the aqueous phase, inducing the formation of nanospheres [13].

\section{- New Technology To Prepare Polymer Nanoparticle}

New preparation method for nanoparticles by modifying the spontaneous emulsification solvent diffusion (SESD) method is developed. In the original SESD method, PLGA poly (DLlactide-co-glycolide) is dissolved in the organic solvent mixture consisting of dichloromethane and acetone, which are a less water-miscible organic solvent and a freely water-miscible organic solvent, respectively. The polymeric solution obtained above is slowly poured into the emulsifier containing aqueous phase with agitating by a stirrer. Nanoparticles are formed via the following steps: when the polymeric solution is added, emulsion droplets are formed in the aqueous phase; acetone quickly diffuses out from each emulsion droplet, drastically reducing its size to nano-order; and the consequent 'solvent-evaporation' process, in which the remaining dichloromethane is removed from the system, makes the droplets solidify to finally form polymeric nanoparticles.

This process seems rational, based on physicochemically interesting phenomena. However, due to a considerable amount of residual dichloromethane, the particles are likely to aggregate during the solvent-evaporation process.

- Modified-SESD method
PLGA nanoparticles are prepared by the modified-SESD method using various solvent systems consisting of two water-miscible organic solvents, in which one solvent has more affinity to PLGA than to PVA and the other has more affinity to PVA than to PLGA. The modified-SESD method provided a good yield of PLGA nanoparticles over a wide range of composition ratios in the binary mixture of organic solvent .In the modified-SESD process, the mixture of two water-miscible organic solvents, such as ethanol: acetone or methanol: acetone was employed, instead of using the mixture of dichloromethane and acetone. This alteration prevented the aggregation of particles even at a high fed amount of polymeric solution, resulting in improvement in yield as acceptable for industrial purposes. This alteration also provided some additional advantages; for instance, the use of a toxic solvent such as dichloromethane could be avoided in the preparation process; the recovering and purifying process could be simplified by using ultrafiltration technique to omit the solvent-evaporation process; and uniform nanoparticle dispersion could always be attained by even mild agitation[14].

\section{Advantages of APD}

- Lower spraying viscosities

- Cost effective

- Higher solids loading

- Higher spray rates

- No solvent environmental, toxicity, or flammability issues

- Reduced energy requirements relative to aqueous polymer solutions[15].

\section{Application and scope of APD}

- Development of polymeric films of APD using novel technologies and optimization of process variables. 
- Development of APD for foods and nutraceuticals.

- Development of new techniques to develop APD like nanotechnologies.

- Development of APD containing blends of polymers and their performance characterization[16].

\section{Conclusion}

Report from various literature shows that aqueous polymeric dispersion (APD) is a better means of coating as compared to conventional or solvent based coating technique. A stable dispersion can be obtained through the optimization of surfactant and stabilizer. APD exhibits superior stability with compared to solvent based coating. The preparation of aqueous dispersion is easy and requires short time which decreases the cost of the coating process. APD offer several advantages over polymers dissolved in organic solvents including lower spraying viscosities, higher solids loading, higher spray rates, no solvent environmental toxicity or flammability issues, and reduced energy requirements relative to aqueous polymer solutions.

\section{References}

[1]. Jones David.Pharmaceutical Application Of Polymers For Drug Delivery.2004;15:3-5

[2]. Raizada Ankita, Bandari Anil, Kumar Brijesh . Polymers In Drug Delivery: A Review, International Journal Of Pharma. Research And Development.2002;2:9-20

[3]. Xinke Caoa, C, Qizhen Gaob, Ping Gaoa, Pingtian Dinga, , Zibin Gaoa, Xiyang Suna. Preparation And Characterization Of A Novel Aqueous Dispersion Of Eudragit E For Coating Dispersions Of Eudragit E For Coating,
Asian Journal Of Pharmaceutical Sciences.2007:2: 29-37

[4]. Pawar Avinash S., Bageshwar Deepak V., Khanvilkar Vineeta V., Kadam Vilasrao J. Advances In Pharmaceutical Coatings, International Journal Of Chemtech Research. 2010;2: 733-737

[5]. Ronald Obei.Aqueous Dispersion Utilizing Corboxyalkyl Cellulose Esters And Water Reducible Polymers,United State Patent

[6]. Mehta Naveen, Gupta Mahesh Kumar, Jain Anurekha. Aqueous Polymeric Dispersion(APD) a Third Generation (3G) Pharmaceutical Coating System,Inventi Journal.2011:103-106

[7]. Ms Mittal N Zala .Techniques For Powder Particle Coating: A Review ,International Journal Of Pharma World Research, 2011;2:1-5

[8]. Yang Zhenzhong, Xu Yuanze, Zhao Delu, Preparation Of Water Based Dispersions Of Epoxy Resin By Phase Inversion Emulsification Technique Study On Rheological Behavior Of Phase Inversion.1998

[9]. Vanderhoff J.W. Inversion Emulsion Polymerization Of Acrylamide: Polymerization Kinetics And Process Development, Journal Of Dispersion Science And Technology. 1984;5:323-363

[10]. Hua Zou, Shishan Wu, And Jian Shen. Polymer/Silica Nanocomposites: Preparation, Characterization, Properties, And Applications, Chem. Rev. 2008;108: 3893-3957

[11]. Valter Castelvetro, Cinzia De Vita, Nanostructured Hybrid Materials From Aqueous Polymer Dispersions. Advances In Colloid And Interface Science. $2004: 167-185$

[12]. Percy M. J. et.al. , Synthesis Of Vinyl Polymer-Silica Colloidal Nanocomposites Via Aqueous Dispersion Polymerization. 2003;19:, 2072-2079

[13]. Allémann E. Preparation Of Aqueouspolymeric Nanodispersions By A Reversible Salting-Out Process: Influence Of Process Parameters On Particle Size, International Journal Of Pharmaceutics. 1992;87: 247-253

[14]. Hideki Murakami, Masao Kobayashi, Hirofumi Takeuchi, Yoshiaki Kawashima. Preparation Of Poly(Dl-Lactide-Co-Glycolide) Nanoparticles By Modified Spontaneous Emulsification Solvent Diffusion Method,International Journal Of Pharmaceutics .1999;187: 143-152

[15]. James W Macginity, Linda A. Felton,Aqueous Polymeric Coatings For Pharmaceutical Dosage Forms, Third Edition. 176:1-47

[16]. Takashi Takayanagis, Masaaki Yamabe, Progress of fluoropolymers on coating applications Development of mineral spirit soluble polymer and aqueous dispersion Progress in Organic Coatings. 2000;40:185-190 